\title{
Estudantes Universitários em Tempos de HIV: $O$ contexto da testagem ${ }^{1}$
}

\author{
Tania Maria Cemin Wagner \\ Alice Maggi \\ Carla Tessari de Souza \\ Ricardo da Silva de Souza \\ Universidade de Caxias do Sul
}

\begin{abstract}
RESUMO
O objetivo do estudo foi conhecer o posicionamento de estudantes universitários diante da decisão de se testarem para o HIV e da possibilidade de participarem em estudos sobre vacina, caracterizando tais estudantes do ponto de vista sociodemográfico. Os participantes foram 645 estudantes matriculados em diferentes cursos de graduação de uma universidade localizada na região sul do Brasil, dentre esses participantes a média de idade era de 25,32 anos, $442(68,5 \%)$ eram do sexo feminino e 202 $(31,3 \%)$ do sexo masculino. Um questionário foi aplicado, com 33 questões, abordando dados sociodemográficos, bem como situações objetivas e hipotéticas, com opções de escolha simples e múltiplas. Os resultados indicaram que $197(30,5 \%)$ dos partiticpantes se testaram para o HIV. Encontrou-se uma associação estatisticamente significativa (Teste Exato de Fisher $=0,000$ ) e uma correlação alta $(0,000)$ entre terem realizado o teste e terem pensado sobre o mesmo A investigação revelou a necessidade dos espaços universitários oferecerem possibilidades de discussão e de campanhas específicas.
\end{abstract}

Palavras-chave: aids; testagem; estudantes universitários.

\section{ABSTRACT \\ Undergraduate Students in Times of HIV: Testing context}

The aim of this study was to better explain the attitudes of university students regarding the decision to undergo HIV testing and the possibility of taking part in studies on vaccinations, by categorizing these students from a socio-demographic viewpoint. The participants were 645 students from different undergraduate courses at a university in the South of Brazil - mean age 25.32 years, $442(68.5 \%)$ were female and $202(31.3 \%)$ were male). A questionnaire was administered, with 33 multiple choice questions, covering socio-demographic data, as well as objective and hypothetical situations. The results clearly showed that 197 (30.5\%) of the participants had been tested for HIV. One met a statistical significant association (Exact of Fisher $=0,000)$ and a high correlation $(0,000)$ between having been tested and to have thoughts on the same. The study revealed the need for university spaces to provide for the possibility of discussions and specific campaigns.

Keywords: aids; testing; undergraduate students.

Esse estudo teve como objetivo conhecer o posicionamento de estudantes universitários diante do Vírus da Imunodeficiência Adquirida (HIV) e, em especial, no que se refere à decisão de se testarem para a identificação do HIV e à possibilidade de participarem de estudos sobre vacinas como voluntários, caracterizando-os também do ponto de vista sociodemográfico. Estimava-se uma possível relação de coerência entre a decisão de se testarem para a presença de HIV e de fazerem parte de um estudo experimental sobre vacina.
Os resultados pretendem contribuir com estudos na área da psicologia até agora pouco realizados no contexto brasileiro sobre esse aspecto da soropositividade, conforme Castro e Remor (2004).

A aids destaca-se entre as enfermidades infecciosas devido à grande magnitude e extensão dos danos causados às populações. As particularidades dessa epidemia dependem, entre outros fatores, do comportamento humano individual e coletivo (Brito, Castilhos \& Szwarcwald, 2000). Devido ao longo período de 
latência clínica, média de onze anos dos sintomas, mesmo que uma vacina cem por cento eficaz fosse desenvolvida e empregada no momento, casos de aids continuariam a ocorrer em grande número nos próximos dez a vinte anos (Rachid \& Schechter, 2001). Como apontam Gabriel, Barbosa e Vianna (2005), em escala mundial, aproximadamente 42 milhões de pessoas, entre homens, mulheres e crianças, vivem com o HIV/aids, sendo que as mulheres, em 2000, representavam $45 \%$ dos infectados. No ano de 2002 , cerca de 5 milhões de pessoas foram infectadas, e o total de óbitos ultrapassou os 3 milhões. Em 2005, em torno de 200.000 pessoas foram infectadas pelo HIV e, aproximadamente, 66.000 pessoas morreram de aids. Entre jovens na faixa de 15 a 24 anos, estima-se que $0,4 \%$ das mulheres e $0,6 \%$ dos homens viviam com HIV em 2005.

O Brasil tem uma epidemia que já se fez presente em todos os seus 26 estados. Embora a prevalência nacional de HIV entre gestantes tenha permanecido abaixo de $0,5 \%$, há um número crescente de novas infecções por HIV entre mulheres, e aquelas que vivem em situações de pobreza parecem correr risco desproporcional de infecção. A epidemia parece estar estabilizada e a prevalência de HIV permanece inalterada $(0,6 \%)$ desde 2000 . Por outro lado, tem-se observado uma tendência de diminuição de incidência de aids na região Sudeste, como também entre homens que fazem sexo com homens (HSH), jovens e usuários de drogas injetáveis (UDI), no país como um todo (Relatório da UNAIDS, 2005, citado por Chequer 2006). É importante lembrar que os dados epidemiológicos se distribuem por continentes e por grupos, nem sempre com contornos perceptíveis, já que por vezes aborda a linha de pobreza, mas em outras aborda hábitos como UDI, que não são privilégio de um ou outro grupo. Segundo informações do RIPSA (2002), desde então não mais se identifica grupos de risco, mas comportamentos e situações de risco e vulnerabilidade, que podem ser praticados por qualquer pessoa, e não só por alguns. São as condições concretas de existência dos indivíduos às condicionantes das situações de vulnerabilidade ou de preservação e de melhoria da saúde das pessoas.

Desde o início dessa epidemia, sabe-se que as políticas públicas no campo da aids incluíram a atuação de psicólogos dentro das equipes multidisciplinares. No contexto de implementação do SUS, a inserção do psicólogo para atuação na saúde se deu pela via da integralidade, com o princípio da atenção integral à saúde (Mazza \& Ipiranga, 2003; Spink \& Matta,
2007). A partir dessa participação, existe uma compreensão própria do psicólogo sobre o sistema (o "espaço") e sua atuação na saúde, necessitando que ele procure uma compreensão mais contextualizada com o campo no qual ele está inserido (SUS), acrescentando conhecimentos relativos ao "espaço" no qual estabelece relações, incluindo o político, segundo Mazza e Ipiranga (2003).

O presente estudo abordou o teste para o HIV junto aos universitários, grupo nem sempre contemplado no campo das políticas de saúde pública, inclusive por pertencerem a um grupo social a priori com mais recursos disponíveis para a saúde. Preocupando-se, então, com um olhar a essa população específica, essa investigação tem como premissa a idéia de que o teste, inserido no aconselhamento, consiste, possivelmente, numa alternativa para a vida. Sabe-se que a implementação de políticas com base em pesquisas, a forte parceria com a sociedade civil, uma abordagem voltada para os direitos humanos, uma política de uso do preservativo, o compromisso político do alto escalão e a alocação de recursos humanos são algumas das questões-chave da resposta brasileira no combate à epidemia (PN-DST/AIDS/Ministério da Saúde, Brasil, 2005, citado por Chequer, 2006). Também importante ressaltar as preocupações de autores como Fagundes Junior (2002) e Nascimento (2007) no que diz respeito às discriminações que ocorrem nas interações sociais e violam os direitos humanos das pessoas portadoras do vírus da aids, muitas vezes com graves consequências para a vida amorosa, social, familiar, profissional e mesmo no cuidado à própria saúde. $\mathrm{O}$ direito a não ser discriminado tem como base o princípio de igualdade, e quem é discriminado pode recorrer a uma assessoria jurídica, gratuitamente, para orientação e encaminhamento judicial.

No Brasil, a incidência de casos entre os heterossexuais foi a que mostrou o maior aumento, influenciando de forma decisiva a expansão da epidemia entre as mulheres. Segundo Rachid e Schechter (2001), a relação heterossexual é a forma de transmissão que mais tem contribuído para a feminilização da epidemia em nosso país. A revisão da literatura indica a presença de um conjunto de conhecimentos já reunidos referente ao assunto do HIV muito mais diante da soropositividade e doença do que propriamente quanto a outras medidas preventivas como testagem e vacina, ultrapassando aqueles já divulgados em larga escala quanto ao uso de preservativos. É por isso que estudos dessa natureza, sublinhando os aspectos psicológicos da doença e da busca de tratamento da infecção, assumem relevância na comunidade científica: 
Como relataram os(as) psicólogos/as, no cotidiano do trabalho em equipe, são muitos os desafios enfrentados para que sejam construídas linguagem e postura ética compartilhada pelos diversos profissionais envolvidos no atendimento ao usuário dos serviços. Portanto, sua construção é parte do processo de discussão das situações vividas no campo, que inclui dificuldades e desafios que podem estar relacionadas a diferentes fatores, dentre os quais os sentimentos presentes na prática cotidiana e valores culturais e morais que precisam ser identificados para que o trabalho seja desenvolvido de forma a atender adequadamente às necessidades da população assistida. (Centro de Referência Técnica em Psicologia e Políticas Públicas - CREPOP, 2008, p. 26)

López (2004) alerta que a maioria dos soropositivos não conhecem seu estado sorológico: não acreditam que tenham a necessidade de testar-se, menosprezam situações de risco que já experimentaram ou mantém uma atitude de negação por medo do estigma e da discriminação. A prática crescente do aconselhamento e testagem voluntária para a presença do HIV são fatores importantes para estimular mudanças nos comportamentos de risco que se traduzirão numa prevenção mais eficaz.

A disponibilidade de assistência e tratamento para os portadores de HIV pode constituir um incentivo relevante para que as pessoas decidam averiguar seu estado sorológico. Deve-se ressaltar que os avanços na prevenção secundária (AZT nas mulheres grávidas) e tratamentos (novas drogas e terapias combinadas) se tornam irrelevantes para os pacientes que desconhecem sua condição de infectados. Compreende-se, portanto, a importância de conhecimentos sobre esse assunto em grupos diferentes da população. Os esforços para se reduzir o crescimento da epidemia têm impacto tanto para os não infectados como para as pessoas que contraíram o vírus e desconhecem sua situação. Frente às limitações de algumas campanhas de prevenção, a maior parte dos infectados procura assistência médica em etapas avançadas da doença, quando as vantagens das terapias precoces são inaplicáveis.

Em relação à bibliografia consultada, foram destacados trabalhos específicos com estudantes de ensino médio e universitários, abordando aspectos altamente relevantes como o impacto da informação preventiva acerca do HIV/aids (Camargo \& Bárbara, 2004), a prática do sexo seguro (Dall'Agnol, 1999) e a constante recomendação de intervenções que, efetivamente, apresentem respostas possíveis aos números crescentes da epidemia no país (Gimenes \& cols, 1996). Em todos os trabalhos fica ressaltada uma integração da universidade com setores de saúde da comunidade, razão pela qual também se justifica o presente estudo. Devido à complexidade do tema, nem sempre uma mesma intervenção preventiva atinge todos os seus objetivos. No estudo de Camargo e Bárbara (2004), por exemplo, o uso de panfletos como instrumento de veiculação da informação preventiva sobre a aids atingiu o impacto desejado no que se refere à informação acerca do HIV/aids. Porém, não obteve o mesmo sucesso quando pretendia alguma modificação quanto ao uso de preservativos entre 300 estudantes do ensino médio de escolas particulares e públicas de cidades de porte médio na região sul do país.

Dall'Agnol (1999) também abordou a prevenção da contaminação pelo HIV na adolescência, buscando conhecer as variáveis implicadas na intenção e solicitação do uso de preservativo, contemplando, respectivamente a especificidade do sexo. A exemplo do estudo anterior, foi também utilizado um questionário construído de acordo com os pressupostos da Teoria da Ação Racional em que foram pesquisados 843 estudantes da capital de um dos estados do sul do Brasil, na faixa etária de 14 a 21 anos, incluindo o final do ensino fundamental, o ensino médio e os primeiros anos do ensino universitário. Por meio da pesquisa, observou-se que o uso e a solicitação do uso de preservativo está ligado às normas subjetivas dos amigos e dos parceiros desses participantes, além de variáveis referentes às experiências sexuais e ao estado civil deles, mostrando ainda uma postura positiva em relação ao uso de preservativo.

No âmbito da intervenção, destaca-se a experiência de um Departamento de Psicologia de uma universidade da região sudeste do país ao constatar junto às autoridades sanitárias que $45,2 \%$ do total de casos de aids notificados naquela região compreendiam a faixa etária de 15 a 29 anos. Em consequência, foram elaboradas ações interdisciplinares abrangendo os interesses da assistência e da formação na área da saúde por meio de atividades de fluxo contínuo e da realização de workshops. Além disso, foram realizados atendimentos individuais, compostos por aconselhamento, encaminhamento e testagem para HIV, bem como os desdobramentos correspondentes (Gimenes \& cols., 1996). Ainda referente à testagem, é fundamental destacar a dificuldade em conhecer a demanda, considerando a existência de serviços públicos e privados nem sempre integrados e a obrigatoriedade da notificação dizer respeito exclusivamente à doença instalada e não à soropositividade. 
As pesquisas com estudantes universitários homogenizam os aspectos socioculturais, minimizando diferenças constatadas quando comparados com outros grupos, conforme indicam os estudos de Peltzer, Nzewvi e Mohan (2004) e de Pinkerton, Wagner-Raphael, Craun e Abramson (2000). Por outro lado, sabe-se de indicação, de cunho mais adequado, quando se aborda características dos grupos que devem fazer parte de estudos relativos à vacina, uma vez que constituem populações de alto risco de infecção e que aderem a complexos protocolos com frequentes testes para HIV. Muitas vezes o cumprimento dessa dupla condição dificulta o reconhecimento do grupo ideal para fazer parte do experimento com vacinas. Já que há muito tempo se superou o conceito de grupo de risco, preferindo-se denominar comportamento de risco, importante contemplar um grupo dos estudantes universitários em estudos dessa natureza. Além do mais, ele constitui preferencialmente o grupo-alvo das campanhas preventivas em termos de faixa etária, principalmente pelo fato de terem intensa vida sexual (D’Amorim, 2002).

Dessa forma, faz-se necessário conhecer o posicionamento de estudantes universitários diante do tema da testagem, não somente se a realizaram ou não, mas também investigar como ela ocorre em termos de tempo, necessidade e outras circunstâncias. Tais resultados fornecem elementos valiosos para melhor conhecer tal população e contribuir também na proposição de políticas que ofereçam uma resposta efetiva ao problema da epidemia.

O estudo pretendeu, portanto, identificar a posição dos estudantes universitários diante do HIV/aids, focalizando a temática e o contexto da testagem e a disponibilidade de colaborar em estudos para vacinas preventivas. Isso tudo com a presença de conhecimentos que a psicologia possui quanto aos cuidados preconizados acerca dessa temática, tanto que segue abaixo depoimentos de Grupo Focal, realizado no Rio de Janeiro, presente do documento publicado pelo CREPOP (2008):

Nessa área da assistência, foi enfatizada a importância do trabalho do psicólogo na promoção da chamada "adesão ao tratamento", visto que esse profissional, "em função do seu olhar diferenciado, voltado para a subjetividade do portador de HIV", pode, "através de uma melhor compreensão da vida desse sujeito, propor e construir estratégias de adesão ao tratamento" ... "garantindo uma qualidade melhor e um tempo maior de vida". (CREPOP, 2008, p. 32)
Ainda em relação à testagem, esse grupo considera que essa não pode ser obrigatória, como destacado na pesquisa divulgada abaixo:

O Grupo Focal no Rio de Janeiro traz uma reflexão ..., indicando como a Psicologia pode contribuir para a construção de uma política de atenção nesse campo [HIV/aids] pautada por uma cuidadosa problematização: "Segundo os participantes do grupo, a ênfase dada ao aconselhamento nessa área de atuação vincula-se a um "desafio ético", tendo em vista a expansão de uma determinada política que prega a testagem em massa. Essa orientação "requer uma reflexão crítica", pois "tende para o moralismo, o controle e o higienismo", através de uma prática assemelhada a uma "polícia sanitária", sendo preciso a construção de uma outra forma de pensar a testagem, não isolada de um contexto, formando uma "vertente que possa promover a conscientização e a cultura do cuidado, através do acompanhamento." (CREPOP, 2008, p. 33)

A resistência e/ou medo de se submeter ao teste podem estar relacionados ao impacto da própria doença. Remor (1999) indica as principais reações emocionais em pacientes portadores do HIV/aids: o choque da doença devido à incapacidade, aos efeitos da medicação e tratamento, isolamento e rejeição; medo de infectar outros e reinfectar-se; perda das capacidades cognitivas, físicas, sociais e laborais; inexistência de cura, devido ao fato de o vírus controlar ou interferir no futuro da vida da pessoa, aos limites impostos pela doença e à rejeição dos demais, por autoculpabilização e recriminação por ter-se exposto ao HIV; raiva e frustração pela incapacidade de superar o vírus, novas e involuntárias restrições de saúde e estilo de vida, e incerteza do futuro; culpa pela possibilidade de ter infectado outros involuntariamente.

Cohen e Faiman (2003) afirmam que a forma com que cada paciente reage ao diagnóstico de aids depende de diversos fatores: de seu momento de vida, de suas experiências anteriores, das informações que recebeu no convívio social, e do ambiente familiar e cultural em que nasceu e cresceu. É comum nos pacientes que recebem esse diagnóstico o surgimento de diversos sentimentos de difícil elaboração como: ansiedade, raiva, medo, angústia, culpa e depressão, permeados pela incerteza e insegurança em relação ao futuro. Conforme Santos, Nascimento e Felipe (2003), existem alguns momentos do processo diagnóstico e do tratamento que podem ser considerados críticos quanto ao aspecto emocional. Dentre esses aspectos estão: o impacto do diagnóstico; a comunicação do mesmo a parceiros e/ou familiares; as dificuldades 
afetivas ou sociais (relações amorosas, trabalho, amigos, exclusão social); o início ou mudança da medicação anti-retroviral; as alterações do estado clínico; o adoecimento ou internação, e as dificuldades emocionais em aderir ao uso dos antirretrovirais.

$\mathrm{O}$ fato de ser transmissível distingue o HIV como uma doença que se inscreve na relação interpessoal. A pesquisa de Lévêque, citada por Vasconcellos, Picard e Ichai (2003), constata uma diminuição da autoestima dos soropositivos em relação ao grupo-controle no tocante às avaliações ligadas ao futuro e aos campos social, profissional e emocional. Essa constatação permite correlacionar o risco de depressão à baixa da autoestima, isto é, à impressão de não corresponder às suas próprias expectativas nos campos considerados. Controlar a observação do tratamento só tem sentido se o sujeito considera sua vida satisfatória ou, pelo menos, encontra razões para ter esperanças.

\section{MÉTODO}

\section{Participantes}

Os 645 estudantes universitários tinham idade variando entre 17 e 56 anos (média $=25,32$ e desvio padrão $=6,98)$, sendo $442(68,5 \%)$ do sexo feminino e $202(31,3 \%)$ do sexo masculino. Foi critério de inclusão nessa amostra o fato de os estudantes universitários aceitarem participar do estudo quando foram convidados, em sua sala de aula. Esses estudantes eram alunos de diversos cursos, de variados centros dos turnos diurno e noturno, representando $3,2 \%$ do total da população acadêmica de uma instituição comunitária da Região Sul do Brasil. Foram abordados, aleatoriamente, alunos de diferentes áreas do conhecimento das Ciências: Humanas, Biológicas e da Saúde, Exatas e Tecnologia, Jurídicas, Contábeis, Econômicas e Administrativas, Filosofia e Educação; dos cursos de Administração, Direito, Psicologia, Pedagogia, Contábeis. Outras informações relevantes sobre os participantes estarão detalhadas nos resultados.

\section{Instrumentos}

Foi utilizado um questionário com 33 questões abordando dados sociodemográficos, bem como situações objetivas e hipotéticas, com opções de escolha simples e múltiplas em relação à testagem, à vacina, ao risco de exposição ao HIV e à notificação de parceiros. Por recomendação ética, em se tratando de aplicações coletivas e com o intuito de garantir o sigilo das informações, elaborou-se três tipos de ques- tionários (A, B e C), modificando-se apenas a ordenação das opções a serem escolhidas em algumas das questões. Nesse estudo foram analisadas as questões relativas à testagem e o contexto em que ela ocorreu, bem como a questão que aborda a vacina, sendo as demais descritas em outros trabalhos futuros.

\section{Procedimentos}

Após a apreciação e aprovação do Comitê de Ética em Pesquisa da instituição, protocolo 045/04 aprovado em 25/11/2004, foi solicitada a aprovação dos Centros Acadêmicos da universidade e a permissão dos professores para a aplicação do questionário nas salas de aulas, randomicamente escolhidas. Foi realizado o treinamento de quatro alunos de uma disciplina do final do curso de Psicologia, que coletaram os dados. Após a pilotagem do questionário, foram readequadas algumas das questões para a realização da coleta propriamente dita. Mediante a assinatura do Termo de Consentimento Livre e Esclarecido, os participantes responderam individualmente o questionário e devolveram-no preenchido diretamente aos aplicadores.

\section{RESULTADOS E DISCUSSÃO}

A população que participou desse estudo incluiu 471 estudantes universitários solteiros $(73,1 \%)$ e 131 casados $(20,3 \%)$, no que diz respeito ao estado civil. Em relação à naturalidade, $507(78,6 \%)$ são da cidade em que se localiza o campus central. Quanto ao período de ingresso na instituição, $77,7 \%$ ingressou entre os anos de 2000 e 2004, encontrando-se, portanto, na fase inicial de sua formação superior, e os demais $22,3 \%$ - ingressaram entre 1980 e 1999. Dos participantes, $200(31 \%)$ referem-se a estudantes da área de Contábeis, Economia e Administração; 148 (22,94\%) da área de Humanas e 134 (20,77\%) da área Jurídica.

Constatou-se que dos 645 participantes, 438 (67,9\%) não realizaram o teste para o HIV e 197 (30,5\%) já se testaram, um índice muito superior quando comparado aos dados registrados pelo Joint United Nations Programme on HIV and AIDS (UNAIDS) (2006), indicando que menos de $10 \%$ da população vivendo com o HIV em países em desenvolvimento têm conhecimento de seu status quanto à soropositividade ao HIV.

Ainda no que diz respeito ao teste, investigaram-se a razão principal pela qual as pessoas fizeram o teste e o tempo de demora dessa testagem em relação à exposição ao risco (desde uma semana até mais de um mês), uma vez que são fatores determinantes na 
obtenção do diagnóstico e definição do tratamento. As seguintes circunstâncias foram identificadas como razão principal para que os 197 participantes realizassem o teste, ressaltando-se que se constatou uma frequência de 203 respostas, ultrapassando, portanto, o número de participantes que fizeram o teste. Isso pode estar revelando uma falta de coerência do grupo em responder de maneira padronizada a todas as respostas, mas mesmo assim é fundamental identificar os motivos elencados por essa população na busca de maior compreensão das variáveis implícitas nesse processo de testagem. Logo, essas 203 razões se distribuem da seguinte forma: $49(24,14 \%)$ por encaminhamento médico; $46(22,66 \%)$ verificaram por curiosidade; $44(21,67 \%)$ por doação de sangue ou órgãos; $33(16,26 \%)$ por exposição à situação de risco; e 19 $(9,36 \%)$ escolheram a alternativa "outras razões" para ter feito o teste. Esses dados no conjunto revelam, por um lado, razões bem objetivas na busca dos universitários em conhecer o seu status quanto à soropositividade e, por outro, um campo que deveria ser melhor explorado na medida em que aproximadamente $10 \%$ não se incluiu em nenhuma das opções oferecidas.

No que se refere ao tempo para realização da testagem, é importante enfatizar que somente $70(35,54 \%)$ dos 197 participantes que referiram terem feito o teste responderam à pergunta em relação ao tempo transcorrido entre a busca da testagem e a exposição ao risco. As respostas estão distribuídas da seguinte forma: $47(67,14 \%)$ revelaram a busca da testagem após um mês de exposição ao risco; 13 (18,57\%) buscaram no período de uma semana a um mês de exposição; e $10(14,28 \%)$ buscaram no prazo de uma semana. Esse dado dificulta a efetivação das recomendações dos protocolos na área da saúde, quando se preocupam com a identificação precoce da soropositividade.

Além do tempo para a realização da testagem, interessou conhecer os motivos do adiamento na procura do teste, ou seja, mais de um mês da exposição ao risco. Novamente há que se considerar o fato de que 91 participantes responderam essa questão, estimando-se que os demais não se incluíram nessa contingência. As respostas mais frequentes em relação ao tempo de demora da testagem estão distribuídas da seguinte maneira: $18(19,78 \%)$ em função de orientação médica; $14(15,38 \%)$ relacionados ao medo; 10 $(10,99 \%)$ por não saberem se poderiam fazer o teste; 7 $(7,69 \%)$ devido à dificuldade de acesso à testagem; e $6(6,59 \%)$ ao constrangimento em solicitar o teste para o HIV. Essas informações denotam que ainda hoje há dificuldades, tanto em relação à informação de uma busca adequada acerca da testagem, quanto ao acesso à testagem propriamente dita, mesmo tratando-se de estudantes universitários, residentes em uma cidade de porte médio, com serviço de referência na área de HIV/aids. Paralelo a isso, tem-se um percentual de 37,36\% (34 respostas) que informaram outros motivos que estariam retardando a procura pela testagem, configurando-se tanto uma limitação do próprio instrumento em não detectar tal sutileza quanto à indisponibilidade de outro instrumento, até o momento, que evidenciasse essa informação. Porém em relação a essa questão, entende-se estar presente também relações possíveis com o temor em obter um diagnóstico de soropositividade e as implicações que isso representa. Isso pode estar sugerindo que outros estudos sejam desenvolvidos, tanto elencando respostas mais específicas relacionadas aos possíveis temores e fantasias, quanto talvez de cunho mais qualitativo, no sentido de realizar entrevista, a partir da qual a pessoa poderá se sentir mais acolhida em poder verbalizar sobre seus temores. Sabe-se que ambas as abordagens metodológicas apresentam aspectos favorecedores e também dificultadores, dependendo principalmente da interpretação do participante e da temática discutida.

Esse questionário abordou também como os estudantes universitários percebem a necessidade de se testarem e se já pensaram sobre essa possibilidade. Quanto à necessidade de se testar, a população ficou dividida, ou seja, $334(51,8 \%)$ participantes responderam que sim e $302(46,8 \%)$ responderam que não. No que diz respeito a ter pensado sobre a possibilidade de se testar, $352(54,6 \%)$ participantes responderam que sim e $282(43,7 \%)$ responderam que não.

Ao analisar simultaneamente as informações entre os participantes terem realizado o teste e considerarem necessário o mesmo, encontrou-se uma associação estatisticamente significativa (Exato de Fisher = $0,034)$ e uma correlação fraca $(0,079)$. De outra parte, no cruzamento das informações quanto aos participantes terem realizado o teste e terem pensado sobre o mesmo, encontrou-se uma associação estatisticamente significativa (Exato de Fisher $=0,000$ ) e uma correlação alta $(0,000)$. Importante ressaltar que isso revela que a decisão de testar-se parece envolver muito mais um posicionamento subjetivo quanto a esse tema, do que somente quando se refere a uma indicação em formato de advertência, ou seja, nem sempre uma informação se transforma em atitude. Essas informações reforçam a necessidade de se investigar cada vez mais o conhecimento e a postura das pessoas frente ao HIV/aids, denotando que ainda há uma compreensão e 
discurso da doença como se fosse uma preocupação relacionada aos outros, considerando-se, assim, que a pessoa não reconhece o risco em suas próprias práticas cotidianas.
As Tabelas 1 e 2 ilustram a distribuição das frequências das respostas dos participantes considerando as duas variáveis simultaneamente.

TABELA 1

Distribuição de Frequências e Percentuais Quanto à Decisão e Necessidade de Testagem ( $n=645)$

\begin{tabular}{lcccc}
\hline & \multicolumn{4}{c}{ Teste } \\
\cline { 2 - 4 } Necessidade de testagem & Sim & Não & Resp. Branco & Total \\
\hline \multirow{2}{*}{ Sim } & 121 & 209 & 4 & $334(51,8)$ \\
& $(33,4)$ & $(62,6)$ & $(4,0)$ & $(100)$ \\
Não & 74 & 222 & 6 & $302(46,8)$ \\
Respostas em branco & $(24,5)$ & $(73,5)$ & $(2,00)$ & $(100)$ \\
Respostas anuladas & 0 & 1 & 0 & $1(0,10)$ \\
\hline Total & 2 & 6 & 0 & $8(1,4)$ \\
\hline
\end{tabular}

TABELA 2

Distribuição de Frequências e Percentuais Quanto à Decisão e Pensamento em Relação à Testagem ( $n=645)$

\begin{tabular}{lcccc}
\hline & \multicolumn{3}{c}{ Teste } & \\
\cline { 2 - 4 } Pensou em testagem & Sim & Não & Resp. Branco & Total \\
\hline \multirow{3}{*}{ Sim } & 145 & 203 & 4 & $352(54,6)$ \\
& $(41,2)$ & $(57,7)$ & $(1,1)$ & $(100)$ \\
Não & 49 & 227 & 6 & $282(43,7)$ \\
Respostas em branco & $(17,4)$ & $(80,5)$ & $(2,1)$ & $(100)$ \\
Respostas anuladas & 1 & 2 & 0 & $3(0,3)$ \\
\hline Total & 2 & 6 & 0 & $8(1,4)$ \\
\hline
\end{tabular}

Pode-se compreender que essa população de estudantes universitários possui um indicador da percepção de risco e consequentemente a necessidade de realização de teste para verificação de soropositividade. Em relação a esse aspecto, há que se retomar o estudo realizado por Melchior e cols. (2006) referente à avaliação da estrutura organizacional da assistência ambulatorial em HIV/aids no Brasil, que gerou um aumento do número de pessoas que se testaram até hoje no país. A especialização do serviço e a experiência clínica têm sido enfatizadas como importantes atributos da qualidade do cuidado médico desde o início da epidemia. O aconselhamento pré/pós-teste alcança altos índices de realização (93,5\% de aconselhamento em pré-teste e $96,3 \%$ em pós-teste). Amplamente divulgada, a implantação do aconselhamento teve grande aporte de recursos para treinamento, elaboração de material específico e divulgação destas publicações.

No que diz respeito à estimativa de participação de voluntários para um estudo sobre vacina contra HIV/aids, constatou-se que $346(53,6 \%)$ participantes concordariam em se submeter a um estudo experimental. Sabe-se que esse índice deve ser relativizado já que se trata de uma situação hipotética e também pode estar relacionado a uma postura moralmente correta, colaborando para o avanço da ciência em relação a essa doença que reconhecidamente mobiliza intensamente todos os envolvidos, aspecto que será melhor discutido a seguir quando se aborda a teoria da dissonância cognitiva. Além disso, importante evidenciar que se constatou que $198(30,7 \%)$ dos partici- 
pantes responderam à alternativa "depende", sinalizando um potencial de participação.

Diante da indagação de como se comportaria essa variável, participação voluntária de estudo sobre vacina, e a decisão de testar-se constatou-se uma ausência de associação e de correlação entre ambas. $\mathrm{O}$ fato de não ter se constatado nem associação e nem correlação significativas entre essas variáveis revela particularidades em relação a essa patologia no que diz respeito a um componente preventivo diante da epidemia do HIV/aids. Tal resultado coincide com as orientações de Spink e Carvalheiro (2002) quanto ao conhecimento das particularidades de determinados grupos dependendo da temática envolvida, permitindo assim o recrutamento de voluntários de forma mais apropriada em caso de testes de vacinas a serem realizados. Os autores também enfatizam a importância de se detalhar a multiplicidade de sentidos atribuída às vacinas num contexto histórico e social. Há que se contemplar, igualmente, os resultados do Projeto Bela Vista, uma das pesquisas realizadas no Brasil, como resultado da iniciativa do UNAIDS em conjunto com o Ministério da Saúde, visando integrar o país no processo de desenvolvimento de uma vacina anti-HIV/aids (Carvalheiro \& Spink, 2006).

Segundo informações no próprio site do Ministério da Saúde (2009), pesquisadores e representantes de governos e da sociedade civil de 23 países lutam para desenvolver uma vacina eficiente contra a doença. São mais de 25 mil voluntários envolvidos em 46 estudos, sendo 21 em fase de testes pré-clínicos e 25 em testes clínicos. No Brasil, desde 2001, estão em andamento oito pesquisas, sendo seis de vacinas terapêuticas e duas de vacinas profiláticas (ou preventivas) - todas em fases iniciais. O País aplicará R $\$ 25$ milhões até 2012 em pesquisas nesse setor. Os investimentos que viabilizarão o avanço em outros campos da saúde pública - serão aplicados na capacitação de recursos humanos, infra-estrutura laboratorial e criação de plataformas tecnológicas. Esse esforço está contemplado nos Plano Nacional de Vacinas anti-HIV, que abrange o período de 2008 a 2012. Isso denota um investimento em vacina por parte do governo e, consequentemente, a necessidade em se conhecer mais acerca dessa temática e suas derivações.

Ao buscar outras relações possíveis entre as variáveis dessa investigação, vale lembrar que $31,8 \%$ dos participantes que referiram realizar o teste, se mostraram favoráveis à vacina. E os que não realizaram o teste, mas se mostraram favoráveis à vacina foram $66,18 \%$ dos participantes. Porém, chama a atenção que $28,58 \%$ fez o teste e se posicionou contra a vacina. Quanto aos participantes que não realizaram o teste, nem concordaram em fazer a vacina tem-se $71,42 \%$. Portanto, pode-se constatar que a posição favorável de realizar a vacina não está diretamente relacionada a ter realizado o teste de HIV. A Tabela 3 apresenta as informações da distribuição de frequências e percentuais relacionados decisão de participar do estudo experimental sobre a vacina em relação à testagem ao HIV.

TABELA 3

Distribuição de Frequências e Percentuais Quanto à Decisão de Participar de Estudo Experimental Sobre Vacina em Relação à Testagem ao HIV ( $n=645)$

\begin{tabular}{lcccc}
\hline \multirow{2}{*}{ Estudo experimental sobre vacina } & \multicolumn{3}{c}{ Teste } & \\
\cline { 2 - 4 } & $\operatorname{Sim}$ & Não & Resp. branco & Total \\
\hline \multirow{3}{*}{ Sim } & 110 & 229 & 7 & $346(53,65)$ \\
& $(31,8)$ & $(66,18)$ & $(2,02)$ & $(100)$ \\
Não & 26 & 65 & 0 & $91(14,1)$ \\
& $(28,58)$ & $(71,42)$ & - & $(100)$ \\
Depende & 56 & 139 & 3 & $198(30,7)$ \\
Não respondeu & $(28,27)$ & $(70,2)$ & $(1,51)$ & $(100)$ \\
& 5 & 5 & 0 & $10(1,55)$ \\
Total & & & & \\
\hline
\end{tabular}

Nota. Através do Teste $\chi^{2}$ com $p=0,560$, verifica-se não haver associação significativa entre Teste e Vacina. 
A partir das informações acima, evidencia-se que os resultados apontaram para uma não coincidência entre o grupo que fez a testagem e que se disporia a experimentar a vacina, revelando a complexidade do assunto e a necessidade de se estudar detalhadamente os possíveis significados nos estudantes em tempos de HIV/aids. Além de registrar a necessidade de se compreender melhor a complexidade desse tema na nossa sociedade, é possível se pensar em várias formas de se explicar a incoerência constatada. Uma delas refere-se à teoria da dissonância cognitiva que, de acordo com Festinger (1975), considera que "podem existir relações dissonantes ou 'incompatíveis' entre elementos cognitivos; a existência de dissonância origina pressões para reduzi-la e para evitar o seu recrudescimeto; as manifestações da operação dessas pressões incluem mudanças de comportamento, mudanças de cognição e exposição circunspecta a novas informações e novas opiniões" (p.36). Ainda em relação a essa teoria, pode-se encontrar situações em que a concordância com alguma afirmativa represente algo socialmente considerado correto, como é o caso de se abordar acerca de testagem e de uma vacina para uma doença que não tem cura, sendo que pode significar, para algumas pessoas, a obrigatoriedade em se posicionar conforme é esperado. Isso também está relacionado ao fato de que, segundo Festinger (1975), “...a dissonância decorre de situações que suscitam a condescendência forçada e que essa dissonância pode ser reduzida mediante a mudança de opinião privada" (p. 114). Caso não ocorra mudança de opinião, pode acontecer “...processos defensivos que rapidamente se estabelecem para impedir que a nova cognição se consolide de forma irrevogável" (p. 127). É importante ressaltar ainda que o fato desse estudo ter abordado os estudantes universitários sem informação prévia de que nessa aula seriam convidados a participarem desse estudo, pode ser compreendido que houve uma exposição involuntária a essa temática da aids. Seguindo o que preconiza a teoria acima descrita, essa solicitação de participação pode ter criado ou aumentado nos participantes uma dissonância cognitiva, se manifestando na forma de respostas sem uma coerência esperada em relação ao que está sendo abordado. Para além dessa compreensão de âmbito teórico, é importante ressaltar que a partir dessa investigação entendese ter identificado a necessidade de os espaços universitários oferecerem possibilidades de discussão e de campanhas específicas a esses estudantes que, mesmo tendo informação, nem sempre apresentam práticas seguras diante do HIV/aids.
Mesmo que as análises estatísticas não tenham se mostrado com resultados significativos, com esse instrumento e esse tamanho de amostra, qualitativamente a informação deve ser abordada com cautela, uma vez que sinaliza a possibilidade dos universitários serem incluídos como participantes em estudos com vacinas. Ainda com relação ao instrumento aplicado e ao tema da vacina, é importante referir que se tratou de uma situação hipotética, apoiado em Laville e Dionne (1999). Do ponto de vista metodológico, tratase de uma variação do questionário, a qual é amplamente utilizada em psicologia, já que propositadamente se oferece ao participante situações com dilemas e/ou problemas de ordem moral, impacto social, entre outros, e cujo objetivo não é saber a resposta certa ou errada, mas como esses grupos se movimentam em relação às situações. No presente estudo realizou-se uma pergunta fechada de escolha simples, ou seja, sem possibilidades de expressão espontânea por parte do participante, ao contrário de estudos como os de Kohlberg $(1980,1984)$, que investigam o julgamento moral por meio de dilemas éticos, e os de Morin (1994), Moscovici (2005) e Spink e Carvalheiro (2002), que estudam as representações sociais por meio de entrevistas. Portanto, em relação à variável vacina, seria indicada uma exploração qualitativa e uma ampliação da investigação quantitativa. Em relação à ampliação, recomenda-se identificar outros grupos potencialmente disponíveis à experimentação de vacina; e qualitativamente, a proposição de grupos de discussão. Evidencia-se, assim, do ponto de vista metodológico, a necessidade de complementar técnicas de investigação quantitativas e qualitativas.

Quanto à coleta de dados, merece destaque o fato de ter sido realizada por alunos do curso de Psicologia, que também são estudantes universitários, e por isso com possibilidade de identificação junto aos participantes, com relação à linguagem e postura durante a aplicação. Esse trabalho se alinha aos estudos de Ayres, Freitas, Santos, Saletti Filho e Franca Jr. (2003), reforçando a importância de trabalhos de prevenção serem desenvolvidos por alunos, na condição de multiplicadores. Outra investigação nessa mesma perspectiva, também com alunos de Psicologia, referese à intervenção preventiva de Gimenes e cols. (1996), integrando o ambiente universitário e o Centro de Orientação e Apoio Sorológico de São Paulo - COAHENFIL/SP. Nele foram organizados workshops motivados pela necessidade de informação sobre aids, bem como a maneira que a doença vem sendo abordada, relacionado ao trabalho feito pela área da saúde e 
o modo de agir diante do problema. Ao final dos mesmos, foi avaliada a eficácia dos resultados através de questionário, constatando que as expectativas foram atendidas diante das estratégias utilizadas.

De acordo com Kornblit, Goménez, Diz, Petracci e Vujosevich (1997), os estudos do tipo descritivo que se conhecem como estudos KABP (estudos CAPConhecimentos, Atitudes e Práticas) são considerados insuficientes para o conhecimento exaustivo em torno dos componentes sociais da aids. Contudo, constituem um primeiro e necessário passo para a investigação que deve logo prosseguir nos desenvolvimentos em profundidade capazes de ser alcançados com técnicas qualitativas.

Portanto, os resultados indicaram particularidades entre os estudantes universitários no que se refere à testagem e à disponibilidade em participar de algum estudo sobre vacina para o HIV, identificando-se de forma significativa que o grupo disponível a participar, hipoteticamente, de estudo experimental com vacina anti-HIV não coincide com aquele que se testou para o HIV.

\section{REFERÊNCIAS}

Ayres, J. R. C. M., Freitas, A. C., Santos, M. A. S., Saletti Filho, H. C., \& Franca Jr., I. (2003). Adolescência e AIDS: Avaliação de uma experiência preventiva entre pares. Interface, $7(12)$, 113-128.

Brito, A. M., Castilhos. E. A., \& Szwarcwald, C. L. (2000). AIDS e infecção pelo HIV no Brasil: Uma epidemia multifacetada. Revista da Sociedade Brasileira de Medicina Tropical, 34(2), 207-217.

Castro, E. K., \& Remor, E. (2004). Aspectos psicossociais e HIV/AIDS: Um estudo bibliométrico (1992-2002) comparativo dos artigos publicados entre Brasil e Espanha. Psicologia: Reflexão e Crítica, 17(2), 243-250.

Camargo, B. V., \& Bárbara, A. (2004). Efeitos de panfletos informativos sobre a AIDS em adolescentes. Psicologia: Teoria e Pesquisa, 20(3), 279-287.

Carvalheiro, J. R., \& Spink, M. J. P. (2006). Estudo sóciocomportamental e determinação da incidencia de infecção por HIV em uma coorte de homens que fazem sexo com homens. Retirado em 01 de novembro de 2006, de www.aids.gov.br/ final/biblioteca/avalia_8/bela_vista/bela_vista.

Centro de Referência Técnica em Psicologia e Políticas Públicas (CREPOP). (2008). Referências técnicas para a atuação do(a) psicólogo(a) nos programas de DST e AIDS. Brasília: Conselho Federal de Psicologia.

Chequer, P. (2006). O desafio de priorizar o HIV/AIDS em países com baixa prevalência. Retirado em 29 de setembro de 2006, de www.aids.gov.br.
Cohen, C., \& Faiman, C. J. S. (2003). AIDS: Ataque ao sistema de defesas psíquicas. Retirado em 17 de novembro de 2006, de www.cremacre.org.br/v1/aidsataq

Dall'Agnol, R. S. (1999). Prevenção da AIDS na adolescência: Um desafio a psicologia contemporânea. Tese de doutorado não-publicada, Pontifícia Universidade Católica do Rio Grande do Sul.

D’Amorim, M. A. (2002). A prevenção da AIDS em universitários. Temas em Psicologia, 10(1), 53-62.

Fagundes Júnior, J. C. P. (2002). Direito à vida privada e à intimidade do portador do HIV: Aspectos constitucionais. São Paulo: Celso Bastos.

Festinger, L. (1975). Teoria da dissonância cognitiva (E. Almeida, Trad.). Rio de Janeiro: Zahar.

Gabriel, R., Barbosa, D. A., \& Vianna L. A. C. (2005). Perfil epidemiológico dos clientes com HIV/AIDS da unidade ambulatorial de hospital escola de grande porte - Município de São Paulo. Revista Latino-Americana de Enfermagem, 13(4), 509-13.

Gimenes, M. G., Pedrazani, E., Basso, A. F. T., Pontes, A. C., Marque, C. R., Souza, D. M. X., Maldonado, D. P. A., Bertuso, E. C., Silva, E. L., Consonni, E. B., Romão, D. C., \& Banhos, E. (1996). A prevenção da AIDS entre estudantes universitários: A resposta da UFSCAR. Temas em Psicologia, 4, 59 -69.

Joint United Nations Programme on HIV and AIDS. (2006). HIV testing and counselling. Retirado em 29 de setembro de 2006, de www.unaids.org/en/Policies/Testingqdefault.asp

Kohlberg, L. (1980). The meaning and measurement of moral development. Trabalho apresentado na Annual Convention of the American Psychological Association, Toronto, Canadá.

Kohlberg, L. (1984). Essays on moral development: Vol. 2. The psychology of moral development. San Francisco: Harper and Row.

Kornblit, A. L., Goménez, L., Diz, A. M. M., Petracci, M., \& Vujosevich, J. (1997). Y el SIDA está entre nosotros. Buenos Aires: Corregidor.

Laville, C., \& Dionne, J. (1999). A construção do saber: Manual de metodologia da pesquisa em ciências humanas. Porto Alegre: Artmed.

López, L. (2004). Se puede prevenir el SIDA? Madrid: Biblioteca Nueva.

Mazza, S. R., \& Ipiranga, A. S. R. (2003). A inserção da psicologia na saúde e a sua inter-relação com o contexto político do SUS. Em S. Nóbrega-Therrien, M. I. Almeida, J. W. O. Lima (Orgs.). Saúde pública: Concepções, práticas e pesquisas (pp. 97-110). Fortaleza: INESP/EDUECE.

Melchior, R., Nemes, M. I. B., Basso, C. R., Castanheira, E. R. L., Britto e Alves, M. T. S., Buchalla, C. M., Donini, A. A., \& Equipe QualiAids (2006). Avaliação da estrutura organizacional da assistência ambulatorial em HIV/AIDS no Brasil. Revista Saúde Pública, 40(1), 143-151.

Ministério da Saúde. (2009). Brasil forma rede de pesquisadores sociocomportamentais na área de vacinas anti-HIV. Retirado 
em 07 de abril de 2009, de http://www.aids.gov.br/main.asp? View $=\{$ E77B47C8-3436-41E0-AC19-E1B215447EB9 $\} \&$ Tea $\mathrm{m}=\&$ params $=$ itemID $=\{3 \mathrm{~A} 671 \mathrm{~A} 54-\mathrm{AD} 40-4 \mathrm{BEF}-\mathrm{B} 860-\mathrm{F} 9 \mathrm{EDC}$ 5711B4B $\} \% 3 B \& U I P a r t U I D=\{$ D90F22DB-05D4-4644-A8F2FAD4803C 8898$\}$

Morin, E. (1994). A deseuropa. Estudos Avançados, 8(21), 43-50.

Moscovici, S. (2005). Representações sociais: Investigações em psicologia social ( $3^{\mathrm{a}}$ ed.). Petrópolis: Vozes.

Nascimento, V. L. V. (2007). Práticas sociais em situação de discriminação no cenário da AIDS: Sobre direitos, demandas e encaminhamentos. Tese de doutorado não-publicada, Pontifícia Universidade Católica de São Paulo.

Peltzer, K., Nzewvi, E., \& Mohan, K. (2004). Attitudes towards HIV-antibody testing and people with aids among university students in India, South Africa and United States. Indian Journal of Medical Sciences, 58(3), 95-108.

Pinkerton, S. D., Wagner-Raphael, L. I., Craun, C. A., \& Abramson, P. R. (2000). A quantitative study of the accuracy of college student's HIV risk estimates. Journal of Applied Biobehavioral Research, 5(1), 1-25.

Rachid, M., \& Schechter, M. (2001). Manual de HIV/AIDS. Rio de Janeiro: Revinter.

Rede Interagencial de Informações para a Saúde (RIPSA). (2002). Indicadores básicos de saúde no Brasil: Conceitos e aplicações. Brasília: Organização Pan-Americana de Saúde.
Remor, E. A. (1999). Abordagem psicológica da AIDS através do enfoque cognitivo-comportamental. Psicologia, Reflexão $e$ Crítica, 12(1), 1-17.

Santos, C. P., Nascimento, V. L., \& Felipe, Y. X. (2003). Aderência ao tratamento anti-retroviral: Resultados preliminares e reflexões da experiência em um serviço universitário Casa da AIDS. Retirado em 20 de agosto de 2005, de www.aidsportugal.com.br/article.php

Spink, M. J. P., \& Carvalheiro, J. R. (2002). Os múltiplos sentidos das vacinas para a AIDS. Psicologia USP, 13(1). Retirado em 02 de maio de 2005, da base de dados SciELO.

Spink, M. J. P., \& Matta, G. (2007). A prática profissional psi na saúde pública: Configurações históricas e desafios contemporâneos. Em M. J. P. Spink (Org.), A psicologia em diálogo com o SUS: Prática profissional e produção acadêmica (pp. 25-51). São Paulo: Casa do Psicólogo.

Vasconcellos, D., Picard, O., \& Ichai, S. C. (2003) Condições psicológicas para a observação das terapias antiretrovirais altamente ativas (HAART). Revista de Psiquiatria, 25(2), 335344.

Recebido: 03/10/2007

Última revisão: 23/09/2009

Aceite final: 19/10/2009

\footnotetext{
Nota:

${ }^{1}$ Agradecimentos: Prof. Dr. Carlos E. Pinent, Sergio Kakuta Kato, Alessandra P. de Azevedo, Fernanda F. Tochetto, Paulo R. Giron e Rafaele F. Amalcaburio.
}

\section{Sobre os autores:}

Tania Maria Cemin Wagner: Psicóloga Pontifícia Universidade Católica do Rio Grande do Sul (1990), mestre e doutora em Psicologia do Desenvolvimento pela Universidade Federal do Rio Grande do Sul. Docente da Universidade de Caxias do Sul.

Alice Maggi: Psicóloga pela Universidade Federal do Rio Grande do Sul (1982), mestre em Psicologia do Desenvolvimento pela Universidade Federal do Rio Grande do Sul (1995), doutora em Psicologia pela Pontifícia Universidade Católica do Rio Grande do Sul (2000). Docente da Universidade de Caxias do Sul.

Carla Tessari de Souza: Integrante do Laboratório de Pesquisa em HIV/AIDS da Universidade de Caxias do Sul.

Ricardo da Silva de Souza: Graduado em Medicina pela Universidade de Caxias do Sul (1990), Master in Health Administration pela Suffolk University (1994), doutor em doenças infecciosas e parasitárias pelo Laboratório de Retrovirologia da Escola Paulista de Medicina da Universidade Federal de São Paulo (2008). Atualmente é chefe do Laboratório de Pesquisa em HIV/aids do Centro de Ciências da Saúde da Universidade de Caxias do Sul.

Endereço para correspondência: Rua Tronca, 1721 - Exposição - 95010-100 Caxias do Sul - RS - Endereço eletrônico: tmcwagne@ucs.br. 